\title{
Performance Analysis of Watermarking on Indian Classical Songs using Lifting Wavelet Transform and Singular Value Decomposition
}

\author{
C. M. Juli Janardhanan \\ Department of ECE \\ Government Engineering College, Wayanad \\ Mananthavady, Kerala, India
}

\author{
C. Sathish Kumar \\ Department of ECE \\ Government Engineering College, Idukki \\ Kerala, India
}

\begin{abstract}
Digital audio watermarking performs the process of embedding a perceptually transparent digital signature carrying a message about the host signal into a host audio signal. A technique of digital audio watermarking using lifting based wavelet transform (LWT) and singular value decomposition (SVD) is applied to watermark Indian classical songs. In order to confirm the security and improve the robustness of the audio watermarking scheme, binary watermark image encrypted using Arnold transform is used. The encrypted watermark bits are embedded into the singular values of the coefficient matrix of LWT low frequency subband through quantization index modulation (QIM). In order to evaluate the performance of the LWT-SVD based audio watermarking method, subjective and objective quality tests are conducted. Investigations are performed using different wavelets and the performance parameters show that Daubechies (Db4) wavelet is more suited for watermarking of Indian classical songs. Robustness of the algorithm is analyzed by including additive white gaussian noise, denoising, and resampling.
\end{abstract}

\section{Keywords}

Audio watermarking, lifting based wavelet transform, singular value decomposition, Arnold transform, quantization index modulation, Indian classical songs.

\section{INTRODUCTION}

Rapid advances in multimedia technology, telecommunication networks, and high speed computing systems have enabled the transmission and distribution of digital multimedia files, easily and efficiently to distant places. Unauthorized copying and distribution of multimedia files have been increased enormously in recent times and there is a need for enforcing copyright protection of digital data.

In order to enforce the intellectual property rights and protect digital audio media from tampering, watermarking has been proposed as an efficient method [1,2]. Audio watermarking is not an easy process because of the sensitivity of human auditory system (HAS). In addition, the amount of data that can be transparently embedded into an audio sequence is considerably lower than the data that can be hidden in image or video sequences, as audio files normally has a dimension less than that of the image or video files.

Digital audio watermarking can be implemented in two ways, in time-domain and transform domain [1, 3]. Transform domain watermarking methods have been observed to be more efficient and robust as compared to time domain techniques. The widely used transforms for audio watermarking are fast Fourier transform (FFT), discrete cosine transform (DCT), and discrete wavelet transform (DWT) [4-7].

It is found that the conventional wavelet transform has very good performance because of its multi-resolution property and perfect reconstruction [6-9]. But the conventional wavelet transform is mainly computed by convolution which results in high computation load. Besides, the generated floating numbers increase storage requirements. As a result, a new wavelet scheme, lifting based Wavelet transform (LWT) has already been proposed for enhanced performance [10, 11]. LWT based method has been reported to provide good performance in the audio watermarking field $[12,13]$.

This paper presents performance analysis of a methodology for audio watermarking using lifting based wavelet transform and singular value decomposition (SVD) [13]. The scheme has been applied for watermarking Indian classical songs. The binary watermark image is encrypted using Arnold transform $[14,15]$ to confirm the security and improve the robustness of the audio watermarking scheme. The scrambled watermark bits are embedded into the singular values of the coefficient matrix of LWT low frequency subband through quantization index modulation (QIM) [14, 16]. Investigations have been performed using different wavelets, and their efficacies have been analyzed. Performance of the algorithm is studied using the measures, normalized cross-correlation (NC), bit error rate (BER), signal to noise ratio (SNR), peak signal to noise ratio (PSNR), mean square error (MSE), and objective difference grade (ODG). It is observed that Daubechies (Db4) wavelet is most suitable for the application. Robustness of the algorithm is established by including additive white gaussian noise (AWGN), denoising, and resampling. Investigations are performed with many clippings of Indian classical songs and the algorithm presents encouraging results.

\section{LIFTING BASED WAVELET TRANSFORM}

Lifting based Wavelet Transform (LWT) is the second generation wavelet which is based on the traditional wavelet. The basic principle behind the lifting based scheme is to decompose the finite impulse response (FIR) filters in wavelet transform into a finite sequence of simple filtering steps. The main feature of the lifting-based DWT scheme is to break up the high-pass and low-pass wavelet filters into a sequence of upper and lower triangular matrices, and convert the filter implementation into banded matrix multiplications. Computation of the upper triangular matrix is known as primal lifting and this is referred to as lifting the low-pass subband with the help of the high-pass subband. Computation 
of the lower triangular matrix is called dual lifting, which is lifting the high-pass subband with the help of the low-pass subband. These two basic lifting steps are called update and predict.

The LWT scheme often requires far fewer computations compared to the convolution based DWT and needs no auxiliary memory due to the fully in-place calculation [10, 11]. Lifting scheme implements reversible integer wavelet and thus perfect reconstruction is possible for loss-less compression.

\section{ARNOLD TRANSFORM}

The Arnold transform rearranges the order of the pixel values in an image to offer security. The Arnold transform change the positions of the pixel values of the original image by using the concept from linear algebra. The result after applying the Arnold transform will be a shuffled image that contains all of the same pixel values of the original image. Each iteration of Arnold transform makes the image chaotic. Arnold transform has a property that the original image will reappear after $n$ iteration, where the periodicity $n$ depends upon the size of the given image. It is only suitable for encrypting $\mathrm{N} \times \mathrm{N}$ images. Arnold transform is an efficient technique for position swapping, and widely applied to image encryption $[14,15]$.

For an $\mathrm{N} \times \mathrm{N}$ image the Arnold transform, is given as [18-19]

$$
\left[\begin{array}{l}
x^{\prime} \\
y^{\prime}
\end{array}\right]=\left[\begin{array}{ll}
1 & 1 \\
1 & 2
\end{array}\right]\left[\begin{array}{l}
x \\
y
\end{array}\right](\bmod N)
$$

where $(\mathrm{x}, \mathrm{y})$ and $\left(\mathrm{x}^{\prime}, \mathrm{y}^{\prime}\right)$ express the pixel co-ordinates of the original and encrypted images, respectively and $\left[\begin{array}{ll}1 & 1 \\ 1 & 2\end{array}\right]$ is the two dimensional Arnold transform matrix.

The encrypted image may be decrypted by applying the inverse of the Arnold matrix and is given as [20]

$$
\left[\begin{array}{l}
x \\
y
\end{array}\right]=\left[\begin{array}{cc}
2 & -1 \\
-1 & 1
\end{array}\right]\left[\begin{array}{l}
x \\
y^{\prime}
\end{array}\right](\bmod N)
$$

where $\left[\begin{array}{rr}2 & -1 \\ -1 & 1\end{array}\right]$ is the inverse of two dimensional Arnold transform matrix.

Let $\mathbf{A}$ denote the two dimensional Arnold transform matrix, $\mathbf{I}(x, y)$ and $\mathbf{I}\left(x^{\prime}, y^{\prime}\right)^{(n)}$ represent pixels in the original image and the encrypted image obtained by performing Arnold transform $n$ times, respectively. Thus, image encryption using $n$ times Arnold transforms can be written as [19]

$$
\mathbf{I}\left(\mathrm{x}^{\prime}, \mathrm{y}^{\prime}\right)^{(\mathrm{k})}=\mathbf{A} \mathbf{I}(\mathrm{x}, \mathrm{y})^{(\mathrm{k}-1)}(\bmod \mathrm{N})
$$

where $\mathrm{k}=1,2, \ldots, \mathrm{n}$, and $\mathbf{I}\left(\mathrm{x}^{\prime}, \mathrm{y}^{\prime}\right)^{(0)}=\mathrm{I}(\mathrm{x}, \mathrm{y})$.

The encrypted image can be decrypted by iteratively calculating the following formula [2]

$$
\mathbf{J}(\mathrm{x}, \mathrm{y})^{(\mathrm{k})}=\mathbf{A}^{-1} \mathbf{J}\left(\mathrm{x}^{\prime}, \mathrm{y}^{\prime}\right)^{(\mathrm{k}-1)}(\bmod \mathrm{N})
$$

where $\mathbf{J}\left(x^{\prime}, y^{\prime}\right)^{(0)}$ is a pixel of the encrypted image, and $\mathrm{J}(\mathrm{x}, \mathrm{y})^{(\mathrm{k})}$ is a decrypted pixel by performing $k$ iterations and $\mathbf{A}^{-1}$ is the inverse of the two dimensional Arnold transform matrix A.

\section{LWT - SVD BASED AUDIO WATERMARKING}

The basic idea in the LWT-SVD based watermarking is timefrequency decomposition of the signal using suitable wavelet and embedding the bits corresponding to the watermark image into the singular value of the wavelet coefficients matrix. The basic scheme of digital audio watermarking using LWT-SVD is depicted in Fig. 1 [13]. Watermarking is done in either low frequency coefficients or high frequency coefficients based on the nature of the signal and applications. The watermark bits are embedded into the singular value of the wavelet coefficients matrix through quantization index modulation (QIM). QIM can achieve tradeoff among the capacity, imperceptibility, and robustness $[16,17]$. In addition it can realize blind extraction.

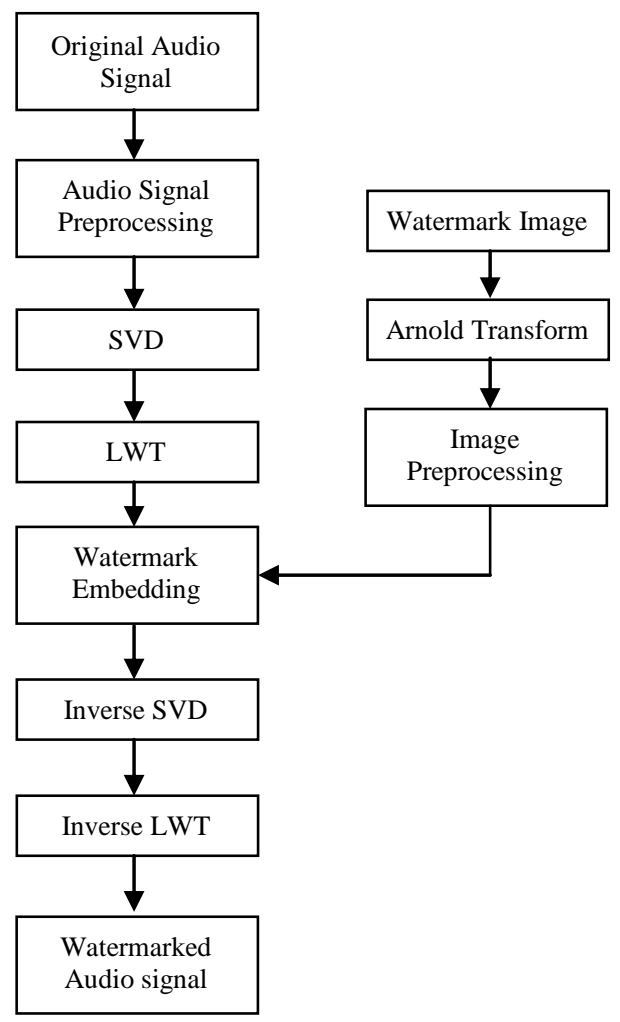

(a) Watermarking embedding

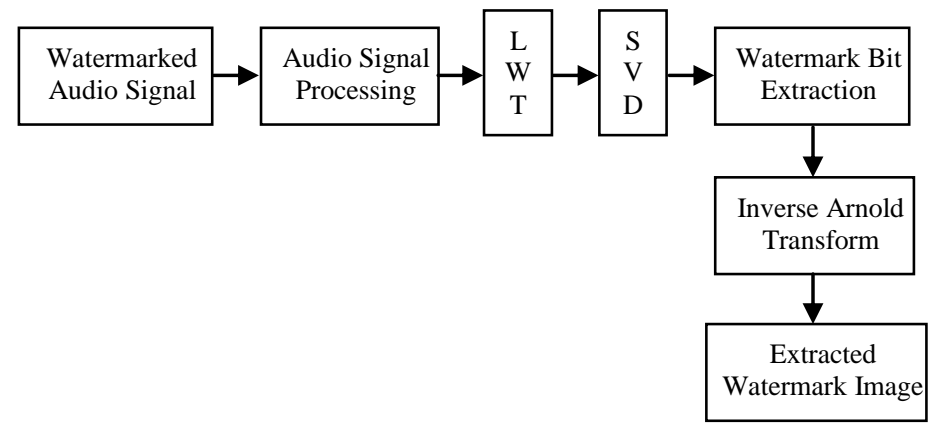

(b) Watermark Extraction

Fig. 1: Basic scheme of digital audio watermarking using LWT-SVD 
SVD is used embedding as changing singular values slightly does not affect the quality of the signal much and the singular values are invariant under common signal processing operations. In order to confirm the security and improve the robustness of the proposed audio watermarking scheme, the watermark should be pre-processed before embedded into the original audio signal. Two-dimensional Arnold transform is chosen to scramble or encrypt image with an aim of increasing the security of the watermark image.

The basic procedures for watermark embedding and extraction can be summarized as

\section{a) Watermark embedding procedure}

1. Binary image watermark is expressed as a two dimensional matrix $(\mathrm{N} \times \mathrm{N})$

2. Scramble the binary watermark image by applying two dimensional Arnold Transform

3. Convert the two-dimensional scrambled watermark image into a one-dimensional watermark vector $W$ of length $N \mathrm{x} N$

4. Decompose the preprocessed audio signal using LWT.

5. Form the non-overlapping blocks using LWT coefficients; form the coefficient matrix

6. Apply the SVD to the coefficient matrix

7. Embed the watermark bit into singular values (SVs) with the QIM method

8. Apply inverse SVD and inverse LWT to obtain the watermarked audio signal.

b) Watermark extraction procedure

1. LWT is applied to the watermarked audio signals.

2. Form the non-overlapping blocks using LWT coefficients; form the coefficient matrix

3. Apply the SVD to the coefficient matrix

4. Extract the watermark bit from the SVs of watermarked audio signal with the QIM method

5. Convert the one-dimensional extracted watermark vector into a two-dimensional extracted watermark image

6. Obtain the final watermark image by descrambling the extracted watermark image with inverse Arnold transform

\section{PERFORMANCE PARAMETERS}

Efficiency of the LWT-SVD based scheme for watermarking Indian classical songs is assessed with the help of a few standard performance parameters [9, 18]. Basically, perceptual quality assessment and watermark similarity detection are used for the evaluating the performance.

\subsection{Perceptual Quality Assessment}

Generally, there are two approaches to perform perceptual quality assessment of audio signals

(i) Subjective listening tests by human acoustic perception.

(ii) Objective evaluation tests

\subsubsection{Subjective Evaluation}

Blind listening tests have been performed for evaluating the subjective quality of the watermarking method. Participants listened to the original and the watermarked audio sequences and were asked to report dissimilarities between them, using a
International Journal of Computer Applications (0975 - 8887)

Volume 83 - No 14, December 2013

5 -grade impairment scale called objective difference grade (ODG) [18] as shown in Table 1.

Table 1: 5-grade impairment scale

\begin{tabular}{|l|c|c|}
\hline \multicolumn{1}{|c|}{ Impairment description } & $\begin{array}{c}\text { ITU-R } \\
\text { Grade }\end{array}$ & ODG \\
\hline Imperceptible & 5.0 & 0.0 \\
\hline Perceptible, but not annoying & 4.0 & -1.0 \\
\hline Slightly annoying & 3.0 & -2.0 \\
\hline Annoying & 2.0 & -3.0 \\
\hline Very annoying & 1.0 & -4.0 \\
\hline
\end{tabular}

\subsubsection{Objective Evaluation}

Objective evaluation is performed by measuring the peak signal to noise ratio (PSNR), signal to ratio (SNR) and mean square error (MSE).

PSNR is defined as

$$
\operatorname{PSNR}(X, \sim X)=20 \log 10 \frac{2^{B}-1}{\sqrt{M S E}}
$$

where $\mathrm{X}$ and ${ }^{\sim} \mathrm{X}$ are the original and the watermarked audio signals, B represents the bits per sample.

SNR is defined as ratio of signal power to noise power and it is given as

$$
\operatorname{SNR}(X, \sim X)=10 \log 10 \frac{\sum_{i=1}^{L} X^{2}}{\sum_{i=1}^{L}(\sim X-X)^{2}}
$$

where $\mathrm{L}$ is the length of the audio signal.

MSE is defined as squared norm of the difference divided by the number of elements in the signal and is given as

$$
\operatorname{MSE}(X, \sim X)=\frac{\| X-\sim X||^{2}}{N}
$$

\subsection{Watermark Similarity Detection}

Normalized cross-correlation (NC) is used to evaluate the similarity between the original watermark and the extracted watermark and is defined as follows [9]:

$$
\begin{aligned}
& N C(W, \sim W)
\end{aligned}
$$

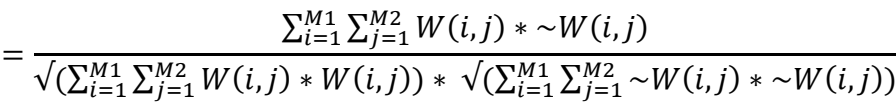

where $\mathrm{W}$ and $\sim \mathrm{W}$ are the original and the extracted watermarks respectively, and $\mathrm{i}, \mathrm{j}$ are indices of the binary watermark image. If $\mathrm{NC}(\mathrm{W}, \sim \mathrm{W})$ is close to 1 , then the similarity between $\mathrm{W}$ and $\sim \mathrm{W}$ is very high. If $\mathrm{NC}(\mathrm{W}, \sim \mathrm{W})$ is close to zero, then the similarity between $\mathrm{W}$ and $\sim \mathrm{W}$ is very low.

The bit error rate (BER) is also used to evaluate the watermark detection accuracy. The BER of the watermarked signal retrieval is defined as follows [9]:

$$
\mathrm{BER}=\frac{\sum_{\mathrm{i}=1}^{\mathrm{M} 1} \sum_{\mathrm{j}=1}^{\mathrm{M} 2} \mathrm{~W}(\mathrm{i}, \mathrm{j}) \oplus \sim \mathrm{W}(\mathrm{i}, \mathrm{j})}{\mathrm{M} 1 * \mathrm{M} 2}
$$




\section{SIMULATION RESULTS AND DISCUSSIONS}

A sequence of experiments has been carried out for careful analysis and comparison of results. The implementation is done using MATLAB 7.11. An image shown in Fig. 2 is used for watermarking and various files of Indian classical songs were used for embedding the watermarks.

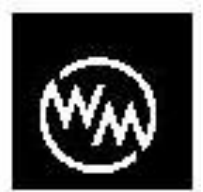

Fig. 2: Watermark image

Two-dimensional Arnold transform is applied to encrypt the image with an aim of increasing the security of the watermark image. Fig. 3 shows the Arnold transformed watermark image with an iteration of 80 .

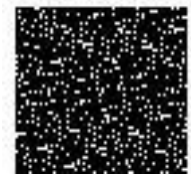

Fig. 3: Arnold transformed watermark image

With the objective of investigating and inferring about the best performing wavelet for the application, LWT decomposition of the audio signal is carried out using Haar, Daubechies and Symlets wavelets. The watermark bits are embedded into the singular value of the approximation coefficients matrix by using QIM method with an intensity of 0.04. Table 2 shows the Normalized cross-correlation (NC), bit error rate (BER), peak signal to noise ratio (PSNR), mean square error (MSE), and objective difference grade (ODG) while using Haar, Daubechies and Symlets wavelets for LWT decomposition.

Table 2: Performance parameters with different wavelets

\begin{tabular}{|c|c|c|c|c|c|c|}
\hline Wavelet & NC & BER & $\begin{array}{c}\text { SNR } \\
(\mathrm{dB})\end{array}$ & $\begin{array}{c}\text { PSNR } \\
(\mathrm{dB})\end{array}$ & MSE & ODG \\
\hline Haar & 1 & 0 & 86.71 & 107.18 & $12 \times 10^{-7}$ & 0.0 \\
\hline Db1 & 1 & 0 & 86.71 & 107.18 & $12 \times 10^{-7}$ & 0.0 \\
\hline Db2 & 1 & 0 & 86.77 & 107.21 & $12 \times 10^{-7}$ & 0.0 \\
\hline Db4 & 1 & 0 & 86.85 & 107.24 & $12 \times 10^{-7}$ & 0.0 \\
\hline Db8 & 1 & 0 & 86.50 & 107.09 & $12 \times 10^{-7}$ & 0.0 \\
\hline Sym2 & 1 & 0 & 86.77 & 107.21 & $12 \times 10^{-7}$ & 0.0 \\
\hline Sym4 & 1 & 0 & 86.83 & 107.24 & $12 \times 10^{-7}$ & 0.0 \\
\hline Sym8 & 1 & 0 & 86.63 & 107.15 & $12 \times 10^{-7}$ & 0.0 \\
\hline
\end{tabular}

It is observed that all wavelets provide similar values of ODG, NC, MSE, and BER. However, based on the values obtained
International Journal of Computer Applications (0975 - 8887)

Volume 83 - No 14, December 2013

for PSNR and SNR, dB4 wavelet is chosen for further investigations.

Watermark is embedded in the audio signal with various watermark intensities. Table 3 shows the performance parameters at different watermark intensities. The watermark is inaudible up to an intensity of 0.1. Considering the results obtained in Tables 2 and 3, Db4 wavelet with embedding at SV of the approximation coefficient matrix using an intensity of 0.05 and 0.1 is chosen for further simulations. Although simulations using only one audio signal have been shown above, it was ascertained that similar results are obtained for other samples of classical songs.

Fig. 4 shows the original audio signal. Fig. 5 and Fig. 6 show the watermarked audio signal and the extracted watermark image (without applying inverse Arnold transform) respectively using Daubechies (Db4) wavelet. Fig. 7 shows final extracted watermark image after applying inverse Arnold transform.

Table 3: Performance parameters for different watermark intensities

\begin{tabular}{|c|c|c|c|c|c|c|}
\hline Intensity & NC & BER & $\begin{array}{c}\text { SNR } \\
(\mathrm{dB})\end{array}$ & $\begin{array}{c}\text { PSNR } \\
(\mathrm{dB})\end{array}$ & MSE & ODG \\
\hline 0.01 & 1 & 0 & 114.1 & 119.0 & 0 & 0.0 \\
\hline 0.03 & 1 & 0 & 92.5 & 109.7 & $0.6 \times 10-6$ & 0.0 \\
\hline 0.05 & 1 & 0 & 82.1 & 105.2 & $2 \times 10-6$ & 0.0 \\
\hline 0.1 & 1 & 0 & 68.6 & 99.3 & $7 \times 10-6$ & 0.0 \\
\hline 0.2 & 1 & 0 & 54.8 & 93.3 & $29 \times 10-6$ & -1.0 \\
\hline 0.3 & 1 & 0 & 46.6 & 89.7 & $68 \times 10-6$ & -2.0 \\
\hline 0.4 & 1 & 0 & 41.2 & 87.4 & $117 \times 10-6$ & -3.0 \\
\hline 0.5 & 1 & 0 & 37.2 & 85.6 & $175 \times 10-6$ & -4.0 \\
\hline
\end{tabular}

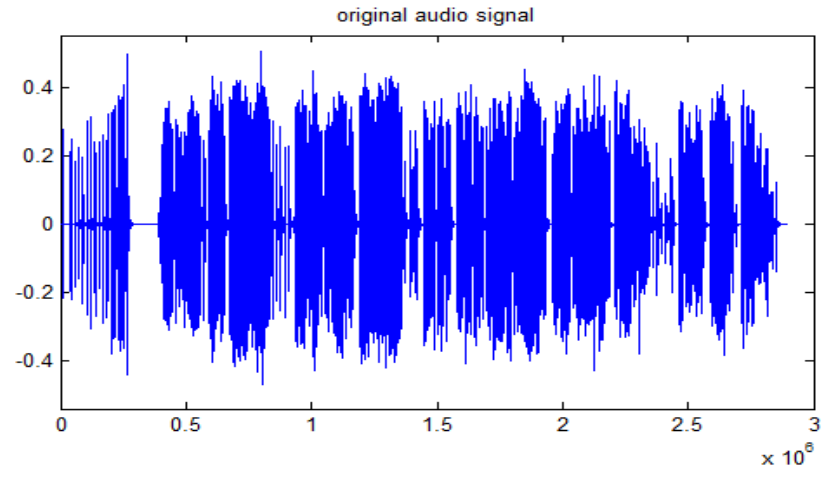

Fig. 4: Original Audio Signal

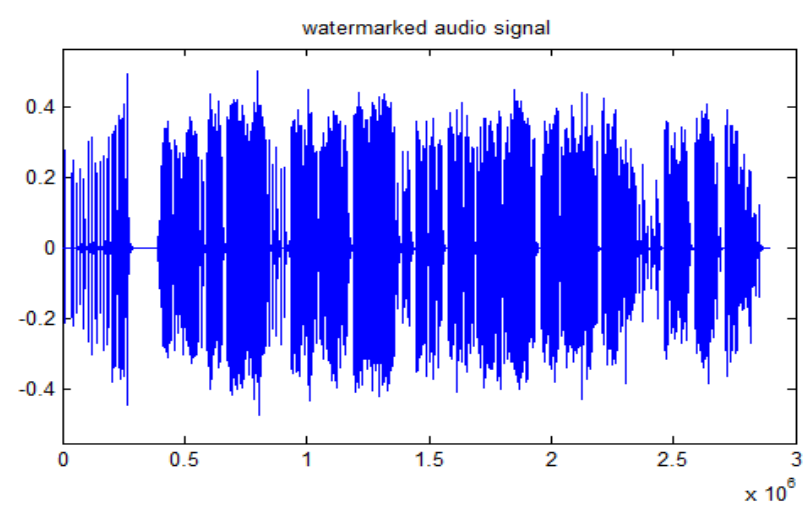

Fig. 5: Watermarked audio signal 


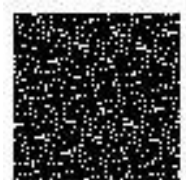

Fig. 6: Extracted watermark image

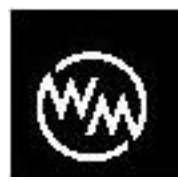

Fig. 7: Final extracted watermark image

\subsection{Analysis of Robustness}

Robustness refers to the ability to detect the watermark after common signal processing operations. Audio watermarking needs to be robust to noise, $\mathrm{A} / \mathrm{D}$ conversion, resampling, etc. [15].

\subsubsection{Effect of $A W G N$ and Denoising}

White Gaussian noise is added to the watermarked signal till the resulting signal has an SNR of $20 \mathrm{~dB}$ and the performance parameters are computed. Table 4 and Table 5 show the performance parameters at different SNR with watermark intensities 0.05 and 0.1 respectively. Fig. 8 shows the extracted watermark from the noised audio signal with noise added with an SNR of $30 \mathrm{~dB}$ with a watermark intensity of 0.05 .

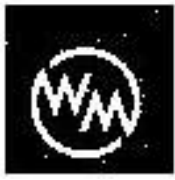

Fig.8: Extracted watermark image with AWGN

Table 4: Performance parameters when AWGN is added with watermark intensity 0.05

\begin{tabular}{|c|c|c|c|c|c|c|}
\hline $\begin{array}{c}\text { SNR } \\
(\mathrm{dB})\end{array}$ & NC & BER & $\begin{array}{c}\text { SNR } \\
(\mathrm{dB})\end{array}$ & $\begin{array}{c}\text { PSNR } \\
(\mathrm{dB})\end{array}$ & MSE & ODG \\
\hline 50 & 1 & 0 & 81.82 & 105.06 & $20 \times 10^{-7}$ & 0.0 \\
\hline 45 & 1 & 0 & 81.06 & 104.73 & $21 \times 10^{-7}$ & 0.0 \\
\hline 40 & 1 & 0 & 79.00 & 103.84 & $26 \times 10^{-7}$ & 0.0 \\
\hline 35 & 1 & 0 & 74.35 & 101.81 & $42 \times 10^{-7}$ & 0.0 \\
\hline 30 & 0.988 & 0.003 & 66.58 & 98.44 & $92 \times 10^{-7}$ & -1.0 \\
\hline 25 & 0.748 & 0.109 & 56.61 & 94.11 & $252 \times 10^{-7}$ & -2.0 \\
\hline 20 & 0.704 & 0.124 & 45.66 & 89.36 & $753 \times 10^{-7}$ & -3.0 \\
\hline
\end{tabular}

International Journal of Computer Applications (0975 - 8887)

Volume 83 - No 14, December 2013

Table 5: Performance parameters when AWGN is added with watermark intensity 0.1

\begin{tabular}{|c|c|c|c|c|c|c|}
\hline $\begin{array}{c}\text { SNR } \\
(\mathrm{dB})\end{array}$ & NC & BER & $\begin{array}{c}\text { SNR } \\
(\mathrm{dB})\end{array}$ & $\begin{array}{c}\text { PSNR } \\
(\mathrm{dB})\end{array}$ & MSE & ODG \\
\hline 50 & 1 & 0 & 68.52 & 99.28 & $76 \times 10^{-7}$ & 0.0 \\
\hline 45 & 1 & 0 & 68.31 & 99.19 & $78 \times 10^{-7}$ & 0.0 \\
\hline 40 & 1 & 0 & 67.68 & 98.92 & $83 \times 10^{-7}$ & 0.0 \\
\hline 35 & 1 & 0 & 65.93 & 98.16 & $99 \times 10^{-7}$ & 0.0 \\
\hline 30 & 1 & 0 & 61.81 & 96.37 & $149 \times 10^{-7}$ & -1.0 \\
\hline 25 & 0.997 & 0.00 & 54.52 & 93.20 & $310 \times 10^{-7}$ & -2.0 \\
\hline 20 & 0.706 & 0.14 & 44.83 & 89.00 & $818 \times 10^{-7}$ & -3.0 \\
\hline
\end{tabular}

Table 6 and Table 7 shows the performance parameters after denoising the watermarked signal with watermark intensities 0.05 and 0.1 respectively.

Table 6: Performance parameters when watermarked signal is denoised with watermark intensity 0.05

\begin{tabular}{|c|c|c|c|c|c|c|}
\hline $\begin{array}{c}\text { SNR } \\
(\mathrm{dB})\end{array}$ & NC & BER & $\begin{array}{c}\text { SNR } \\
(\mathrm{dB})\end{array}$ & $\begin{array}{c}\text { PSNR } \\
(\mathrm{dB})\end{array}$ & MSE & ODG \\
\hline 50 & 1 & 0 & 81.58 & 104.95 & $20 \times 10^{-7}$ & 0.0 \\
\hline 45 & 1 & 0 & 81.14 & 104.76 & $21 \times 10^{-7}$ & 0.0 \\
\hline 40 & 1 & 0 & 80.02 & 104.28 & $24 \times 10^{-7}$ & 0.0 \\
\hline 35 & 1 & 0 & 77.18 & 103.04 & $32 \times 10^{-7}$ & 0.0 \\
\hline 30 & 0.996 & 0.000 & 71.45 & 100.55 & $57 \times 10^{-7}$ & -1.0 \\
\hline 25 & 0.763 & 0.091 & 62.73 & 96.77 & $136 \times 10^{-7}$ & -2.0 \\
\hline 20 & 0.755 & 0.104 & 52.32 & 92.25 & $386 \times 10^{-7}$ & -3.0 \\
\hline
\end{tabular}

Table 7: Performance parameters when watermarked signal is denoised with watermark intensity 0.1

\begin{tabular}{|c|c|c|c|c|c|c|}
\hline $\begin{array}{c}\text { SNR } \\
(\mathrm{dB})\end{array}$ & NC & BER & $\begin{array}{c}\text { SNR } \\
(\mathrm{dB})\end{array}$ & $\begin{array}{c}\text { PSNR } \\
(\mathrm{dB})\end{array}$ & MSE & ODG \\
\hline 50 & 1 & 0 & 68.47 & 99.26 & $76 \times 10^{-7}$ & 0.0 \\
\hline 45 & 1 & 0 & 68.36 & 99.21 & $77 \times 10^{-7}$ & 0.0 \\
\hline 40 & 1 & 0 & 68.03 & 99.07 & $80 \times 10^{-7}$ & 0.0 \\
\hline 35 & 1 & 0 & 67.07 & 98.65 & $88 \times 10^{-7}$ & 0.0 \\
\hline 30 & 1 & 0 & 64.54 & 97.56 & $114 \times 10^{-7}$ & -1.0 \\
\hline 25 & 0.998 & 0.00 & 59.24 & 95.25 & $193 \times 10^{-7}$ & -2.0 \\
\hline 20 & 0.706 & 0.14 & 50.86 & 91.61 & $447 \times 10^{-7}$ & -3.0 \\
\hline
\end{tabular}


Fig. 9 shows the extracted watermark after denoising the watermarked audio signal with noise added with an SNR of $30 \mathrm{~dB}$ with a watermark intensity of 0.05 .

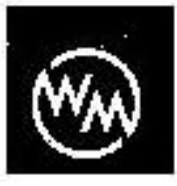

Fig. 9: Extracted watermark image with denoising

\subsubsection{Effect of Re-sampling}

The watermarked signal, originally sampled at $44100 \mathrm{~Hz}$, is resampled at $5500 \mathrm{~Hz}, 11000 \mathrm{~Hz}$, and $22000 \mathrm{~Hz}$ and then restored back by sampling again at $44.1 \mathrm{kHz}$. Table 8 shows the performance parameters after re-sampling the watermarked signal with watermark intensities 0.05 and 0.1 respectively. In both cases, $\mathrm{NC}$, BER, and ODG were obtained as 1,0 , and 0 respectively.

Table 8: Performance parameters with re-sampling with watermark intensity 0.05 and 0.1

\begin{tabular}{|c|c|c|c|c|}
\hline $\begin{array}{c}\text { Watermark } \\
\text { intensity }\end{array}$ & $\begin{array}{c}\text { Resampling } \\
\text { rate }(\mathrm{Hz})\end{array}$ & $\begin{array}{c}\text { SNR } \\
(\mathrm{dB})\end{array}$ & $\begin{array}{c}\text { PSNR } \\
(\mathrm{dB})\end{array}$ & MSE \\
\hline \multirow{3}{*}{0.05} & 22000 & 82.18 & 105.2 & $19 \times 10^{-7}$ \\
\cline { 2 - 5 } & 11000 & 82.18 & 105.2 & $19 \times 10^{-7}$ \\
\cline { 2 - 5 } & 5500 & 82.18 & 105.2 & $19 \times 10^{-7}$ \\
\hline \multirow{3}{*}{0.1} & 22000 & 68.62 & 99.33 & $75 \times 10^{-7}$ \\
\cline { 2 - 5 } & 11000 & 68.62 & 99.33 & $75 \times 10^{-7}$ \\
\cline { 2 - 5 } & 5500 & 68.62 & 99.33 & $75 \times 10^{-7}$ \\
\hline
\end{tabular}

\subsection{Performance Analysis for Different Classical Songs}

In order to establish that the proposed scheme of watermarking based on LWT-SVD through QIM method with watermark image encrypted using Arnold transform is most suitable for Indian classical songs, investigations were further carried out with many such songs and very encouraging results were obtained. Results obtained in case of another 3 such songs are presented in Table 9.

Table 9: Performance parameters when watermarking was applied to 3 different classical songs

\begin{tabular}{|c|c|c|c|c|c|c|}
\hline Song & NC & BER & $\begin{array}{c}\text { SNR } \\
(\mathrm{dB})\end{array}$ & $\begin{array}{c}\text { PSNR } \\
(\mathrm{dB})\end{array}$ & MSE & ODG \\
\hline S1 & 1 & 0 & 96.32 & 106.41 & $14.8 \times 10^{-7}$ & 0.0 \\
\hline S2 & 1 & 0 & 93.21 & 106.55 & $14.3 \times 10^{-7}$ & 0.0 \\
\hline S3 & 1 & 0 & 94.26 & 106.59 & $14.2 \times 10^{-7}$ & 0.0 \\
\hline
\end{tabular}

Robustness was also verified with Indian classical songs by adding AWGN. Table 10 shows the performance parameters when AWGN was added with an SNR of $30 \mathrm{~dB}$ and it
International Journal of Computer Applications (0975 - 8887)

Volume 83 - No 14, December 2013

demonstrate that the watermarking technique is robust against AWGN. Further, the AWGN was removed using denoising techniques and the performance parameters were estimated and the performance is depicted in Table 11 .

Table 10: Performance parameters with AWGN noise of 30dB

\begin{tabular}{|c|c|c|c|c|c|c|}
\hline Song & NC & BER & $\begin{array}{c}\text { SNR } \\
(\mathrm{dB})\end{array}$ & $\begin{array}{c}\text { PSNR } \\
(\mathrm{dB})\end{array}$ & MSE & ODG \\
\hline S1 & 0.996 & 0.000 & 58.36 & 89.93 & $66 \times 10^{-6}$ & 0.0 \\
\hline S2 & 0.986 & 0.003 & 53.72 & 89.40 & $74 \times 10^{-6}$ & 0.0 \\
\hline S3 & 0.979 & 0.005 & 54.22 & 89.20 & $78 \times 10^{-6}$ & 0.0 \\
\hline
\end{tabular}

Table 11: Performance parameters after removing AWGN with denoising

\begin{tabular}{|c|c|c|c|c|c|c|}
\hline Song & NC & BER & $\begin{array}{c}\text { SNR } \\
(\mathrm{dB})\end{array}$ & $\begin{array}{c}\text { PSNR } \\
(\mathrm{dB})\end{array}$ & MSE & ODG \\
\hline S1 & 0.999 & 0.000 & 78.70 & 98.76 & $86 \times 10^{-7}$ & 0.0 \\
\hline S2 & 0.996 & 0.000 & 78.00 & 99.94 & $65 \times 10^{-7}$ & 0.0 \\
\hline S3 & 0.993 & 0.002 & 78.25 & 99.63 & $70 \times 10^{-7}$ & 0.0 \\
\hline
\end{tabular}

\section{CONCLUSIONS}

In this paper, a blind audio watermarking algorithm based on Lifting Wavelet Transform and Singular Value Decomposition, with watermarking image encrypted using Arnold transform, through QIM method is investigated for watermarking Indian classical songs. Simulations using different wavelets and other parameters reveal that watermarking the wavelet approximation coefficients with Daubechies wavelet, $\mathrm{Db} 4$ give best results. Comparison of performances is carried out using the parameters, signal to noise ratio, peak signal to noise ratio, normalized crosscorrelation, bit error rate, mean square error, and objective difference grade. Robustness of the algorithm is also ascertained by incorporating AWGN, denoising, and resampling. The watermarking scheme is observed to provide promising results for Indian classical songs.

\section{REFERENCES}

[1] Cvejic, N. 2004. Algorithms for Audio Watermarking and Steganography. Academic Dissertation, University of Oulu.

[2] Alsalami, M. A. T., and Al-Akaidi, M. M. 2003. Digital Audio Watermarking: Survey. Proc. $17^{\text {th }}$ European Simulation Multi-conference, SCS Europe BVBA.

[3] Bassia, P., Pitas, I, and Nikolaidis, N., 2001. Robust audio watermarking in the time domain. IEEE Trans. Multimedia 3(2), 232-241.

[4] Al-Haj, A., Mohammad, A., and Bata, L. 2011. DWT Based Audio Watermarking, The International Arab Journal of Information Technology, Vol. 8, No. 3, 326333.

[5] Vieru, R., Tahboub R. Constantinescu, C., and Lazarescu, V., 2005. New Results Using the Audio 
Watermarking Based on Wavelet Transform, International Symposium on Signals, Circuits and Systems, ISSCS, IEEE, 441-444.

[6] Datta, K. and Sengupta, I. 2010. A Redundant Audio Watermarking Technique using Discrete Wavelet Transformation, Second International Conference on Communication Software and Networks, 27-31.

[7] Lei B., Y., Soon, I. Y., and Li, Z. 2011. Blind and robust audio watermarking scheme based on SVD-DCT, Signal Processing, 91(8), 1973-1984

[8] Baoyuan, C., Yanli, H., Ruigang, L., and Gang, H. 2011 The audio watermarking system based on wavelet transform algorithm, Cross Strait Quad-Regional Radio Science and Wireless Technology Conference, 1274 1277.

[9] Bhat, K. V., Sengupta, I., and Das, A. 2010. An adaptive audio watermarking based on the singular value decomposition in the wavelet domain, Digital Signal Processing, 20, 1547-1558.

[10] Daubechies, I., and Sweldens, W. 1998. Factoring Wavelet Transforms into Lifting Steps, J. Fourier Anal. Appl., 4(3), 245-267.

[11] Acharya, T., and Chakrabarti, C. 2006. A Survey on Lifting-based Discrete Wavelet Transform Architectures, Journal of VLSI Signal Processing, 42, 321-339.

[12] Lei, B., Soon, I. Y. , Zhou, F., Li, Z., and Lei, H. 2012. A robust audio watermarking scheme based on lifting wavelet transform and singular value decomposition, Signal Processing, 92, 1985-2001.

[13] Erçelebi, E., and Batakçı, L. 2009. Audio watermarking scheme based on embedding strategy in low frequency components with a binary image, Digital Signal Processing 19, 265-277.

[14] Wang, X., Wang, P., Zhang, Z., Xu, S., and Yang, H., 2013. A norm-space, adaptive, and blind audio watermarking algorithm by discrete wavelet transform. Signal Processing 93(4): 913-922.

[15] Tang, Z., Zhang, X. 2011. Secure image encryption without size limitation using Arnold transform and random strategies. Journal of Multimedia, Vol. 6, No. 2 , 202-206.

[16] Chen, B., and Wornell, G. W., 2001. Quantization index modulation: A class of provably good methods for digital watermarking and information embedding. IEEE Transaction on Information Theory, Vol. 47, No. 4 $1423-1443$

[17] Chen, B., and Wornell, G. W., 2001. Quantization Index Modulation Methods for Digital Watermarking and Information Embedding of Multimedia, Journal of VLS Signal Processing, 27, 7-33.

[18] International Telecommunications Union Recommendation. 1998. Method for Objective Measurements of Perceived Audio Quality, BS.1387.

[19] Yang S., Tan W., Chen Y., and Ma W., 2010 Quantization-Based Digital Audio Watermarking in Discrete Fourier Transform Domain, Journal of Multimedia, Vol. 5, No. 2, 151-158. 